\title{
BIG DATA AND INTELLIGENT DECISION METHODS IN ECONOMY, INNOVATION AND SUSTAINABLE DEVELOPMENT
}

\author{
Deng-Feng $\mathrm{LI}^{1 *}$, Pei-De LIU ${ }^{2}$ \\ ${ }^{1}$ School of Management and Economics, University of Electronic Science and Technology \\ of China, Chengdu, 611731 Sichuan, China \\ ${ }^{2}$ School of Management Science and Engineering, Shandong University of Finance and Economics, \\ Jinan, 250014 Shandong, China
}

With the development of computer technology, Internet, the Internet of things, 5G, artificial intelligence, and economic globalization, research modes and means in economy, management, business, innovation, and social science have been demand to change from traditional qualitative and quantitative methods to big data analysis and intelligent decision methods, such as fuzzy cognition, data mining, correlation/association analysis, fuzzy reasoning, evidence theory, multiple agent modeling, artificial intelligence modeling, intelligent computing, Heuristic algorithm, computational experiment method, clustering analysis, neural network analysis, deep learning, and so on. Nowadays, instead of traditional decision methods and modes, the data-driven decision paradigm and intelligent decision making have become prevalent in multidisciplinary and various application fields. Particularly, big data and intelligent decision methods in economy, innovation, business, management, and sustainable development have become promising in the past several years and provide a variety of techniques to the development of economy, innovation, business, management, and sustainable development. This special issue of Technological and Economic Development of Economy entitled "Big Data and Intelligent Decision Methods in Economy, Innovation and Sustainable Development" focuses on the up-to-date modeling, analysis, computation, and synthesis algorithms and approaches concerning big data and intelligent decision making problems, including theoretical, empirical, and experimental works related to big data and intelligent decision methods and associated applications in economy, innovation, business, management, and sustainable development.

The aim of this special issue is to present high-quality papers for promoting novel big data and intelligent decision methods to the research of economy, innovation, business, management, and sustainable development. After the reviewing process according to the aims and

${ }^{\star}$ Corresponding author. E-mail: lidengfeng@uestc.edu.cn 
scope of Technological and Economic Development of Economy and criterion of this special issue, six papers have been selected for publication in this special issue. These papers concern different topics of big data and intelligent decision methods in the research fields of economy, innovation, business, management, and sustainable development. The main contents of the six papers are briefly explained and summarized as follows.

\section{About the papers in this special issue}

The first paper entitled "Forecasting Financial Cycles: Can Big Data Help?" by Marinko Skare and Malgorzata Porada-Rochon, studies the financial cycles based on the financial big data of the UK, USA, Japan, and China for a period 2004 to 2019 by using the singular spectral analysis (SSA) and multichannel singular spectral analysis (MSSA). Their study shows that using financial big data can significantly improve the forecast accuracy for financial cycle components such as residential property prices, credit to private non-financial sector, and credit share in the GDP. The authors confirm that financial big data not only are an important element for studying financial cycles, but also help to better understand the mechanism behind financial cycles, methods and tools for the monitoring and forecasting as well as bring important insight to the policy makers, financial practitioners, academic community and interested in monitoring and studying the financial cycles and their role in financial crisis and thus business cycles.

The next paper entitled "Evaluating the Comprehensive Impacts of Tourism in Hainan by Integrating Input-Output Model with MCDM Methods", written by Ping-Ping Lin, DengFeng Li, Bin-Qian Jiang, Gao-Feng Yu, and An-Peng Wei, proposes a comprehensive evaluation approach through integrating the input-output (IO) model with four multi-criteria decision making (MCDM) methods such as the weighted sum method (WSM), technique for order of preference by similarity to ideal solution (TOPSIS), elimination and choice translating reality (ELECTRE), and preference ranking organization method for enrichment evaluation (PROMETHEE). The developed approach is used to evaluate the comprehensive impacts of tourism sectors on economy, environment, society, and culture in Hainan based on the database of Hainan 2002, 2007, and 2012 IO tables. The empirical study of Hainan's tourism considers four dimensions such as economic contribution, environment pollution, social welfare, and cultural impacts from tourist activities in Hainan. The results show that economic dimension is the most important consideration and the overall performance of tourism in Hainan has a trend of first increasing and then decreasing from 2002 to 2012. Their findings can offer insightful policies for the development of Hainan's tourism. The authors point out that speeding up the integration between the tourism and culture sectors is an important and a main content for Hainan's tourism development in the future and the green tourism is another future direction.

In the third paper entitled “Topological Structural Analysis of China's New Energy Stock Market: A Multi-dimensional Data Network Perspective”, Pei-De Liu, Ke-Dong Yin, Zhe Liu, and Chong Huang investigate the topological structure of China's new energy stock market based on the random vector (RV) coefficient network using the daily prices of 60 component stocks of CSI (China Stock Index) New Energy Index spanning the period January 4, 2012 to 
March 29, 2019. The RV coefficient, instead of the Pearson correlation coefficient, can better reflect the similarity between stocks from the perspective of multi-dimensional data, including the opening price, maximum price, minimum price, and closing price of stocks. Their study shows that the scale-free characteristics of China's new energy market stock network are not significant and the most influential stock in the new energy sector is Hengdian Group DMEGC Magnetics Co., Ltd., which has the most connections with other stocks, plays an important information hub in network information transmission, and has the strongest ability to use other stocks for information transmission. Their findings not only help to understand the interaction between Chinese new energy stocks and the pricing mechanism of stocks, but also pay attention to the new energy storage industry and optimize investment portfolios according to the influence assessment of stocks and sub-sectors.

The fourth paper entitled "A General Multi-Attribute Multi-Scale Decision Making Method based on Dynamic LINMAP for Property Perceived Service Quality Evaluation", written by Wen-Jin Zuo, Deng-Feng Li, and Gao-Feng Yu, develops a general multi-attribute multi-scale (MAMS) method based on the dynamic linear programming technique for multidimensional analysis of preference (LINMAP) for evaluating the property perceived service quality (PPSQ) with multi-stage, multi-source and large-group perceived information, which may be regarded as a kind of dynamic group multi-attribute decision making problems with heterogeneous and large-scale data. The empirical study of the PPSQ evaluation shows that the developed method not only enriches the PPSQ evaluation methods, but also seems to be suitable for complex scale-data processing in modern service. Their developed method can provide a new way to deal with dynamic heterogeneous multi-attribute group decision making problems with large-scale data and incomplete information.

In the fifth paper entitled "Convergence Analysis of Environmental Efficiency from the Perspective of Environmental Regulation: Evidence from China”, Guo-Xiang Li, Su-Ling Feng, Hao-Yue $\mathrm{Wu}, \mathrm{Li}$-Ping Li, and Wen-Ting Zhou investigate the regional heterogeneity of environmental efficiency convergence and explore the impact of environmental regulation on the environmental efficiency convergence through constructing the fixed effect model and threshold regression model based on the Malmquist index and data envelopment analysis (DEA). Empirical analysis shows that the differences in environmental efficiency have a convergence trend in China as well as in the eastern, central, and western regions. The authors point out that research and development $(R \& D)$ investment and outward foreign direct investment (OFDI) have a significant positive effect on environmental efficiency under environmental regulation and help to narrow the environmental efficiency gap between regions.

Finally, in the paper entitled "Research on the Evolution of Innovation Behavior of New Generation Entrepreneurs in Different Scenarios”, Ai-Wu Zhao, Zhen-Zhen Sun, Hong-Jun Guan, and Jing-Yuan Jia construct heterogeneous agents with different individual attributes (e.g., risk preference, innovative spirit, innovative ability, and cognitive social capital) and resources (e.g., inherent capital, talent, and technology resources), and maps the decision making mechanism to agents' behavior rules. Moreover, the authors explore the dynamic innovation process of new generation entrepreneurs in different structural social capital and different policy environment by using simulation and computational experiment methods. Experimental results show that the capital, technology, and talent conditions are very impor- 
tant for innovation implementation although internal elements such as individual innovative spirit, innovative ability, and cognition of social capital determine the innovation intention. Their study helps to reduce the negative impact of innovation risk on continuous innovation and establish risk guarantee mechanism to promote the innovation of new generation entrepreneurs.

\section{Acknowledgements}

We would like to thank the three Editor-in-Chief of Technological and Economic Development of Economy, Professor Edmundas Kazimieras Zavadskas, Professor Jonas Šaparauskas, and Professor Zenonas Turskis for their great support and kind help to achieve this special issue. We would also like to acknowledge all the reviewers for their support in the evaluation of the papers submitted to this special issue and the contributors. 